which could not be separated. Diphenylmethane and fluorene give a mixture of chloro-derivatives, respectively, which could not be separated and isolated and in these cases no nitration takes place. In the case of naphthalene it has been found that no chlorination at all takes place, but the naphthalene is nitrated. Cymene, styrene and tetrahydronaphthalene are nitrated and chlorinated. Hydrocarbons which undergo decomposition under the influence of aqua regia, such as cymene, styrene, amylene, diisobutylene, triisobutylene, give chloropicrin which is generally a product of such decompositions, as will be shown later on. Cyclohexane, on account of its volatile nature, could not be chlorinated by means of aqua regia, whereas dimethylcyclohexane is simultaneously nitrated and chlorinated.

Calcutta, India.

[Contribution from the Chemical Laboratory of the Presidency Coliege.]

\title{
HALOGENATION. XII. THE FORMATION OF CHLOROPICRIN AND TETRACHLOROQUINONE BY THE ACTION OF AQUA REGIA ON ORGANIC SUBSTANCES.
}

by Rasik lal Datta and Niear Ranjan Chatterjee.

Received June 17, 1916.

In a previous communication ${ }^{1}$ it has been shown that chloropicrin is formed by the action of aqua regia on several organic substances. It has now been found that whenever an organic compound breaks up destructively under the influence of aqua regia, chloropicrin is invariably produced. The chloropicrin formed is sometimes accompanied by very small quantities of other chloronitroderivatives of methane. In the case of certain aromatic compounds, tetrachloroquinone is formed as the end product of the action of aqua regia on them. As tetrachloroquinone itself breaks up to chloropicrin under the influence of aqua regia, the latter is also formed under such decompositions. The formation of chloropicrin has been noticed only in isolated cases by previous workers.

Stenhouse ${ }^{2}$ showed that chloropicrin is formed by the distillation of nitro compounds with bleaching powder solution. Kekules obtained it by the distillation of alcohol with sodium chloride, potassium nitrate and sulfuric acid. Later on, he again prepared the substance by the action of strong nitric acid on chloral. ${ }^{4}$ The action of the same reagent on chloroform was found to give chloropicrin. ${ }^{5}$ Levy and Jedicka ${ }^{6}$

${ }_{1}$ Datta and Chatterjee, This Journal, 37, 567 (I915).

${ }^{2}$ Ann., 66, 24I (I 848 ).

${ }^{3}$ Ibid., I0I, 212 (1857).

4 Ibid., 106, I44 (1858).

${ }^{5}$ Mills, Ibid., I60, II 7 (I871).

- Ann., 249, 86 (1888). 
showed that this can be obtained by the action of chlorine on nitranilic acid. Hofman ${ }^{1}$ gave a method for the preparation of this compound by the action of bleaching powder on picric acid.

On examining the above modes of formation, it will be noticed that they fall into two classes. The first is the distillation of nitro compounds with bleaching powder and the second is the action of nitric acid on halogenated organic compounds containing at least three chlorine atoms. The first mode requires that the decomposing substance must have a nitro group, while the second requires the presence of three chlorine atoms, these representing the two kinds of groups attached to the carbon atoms in chloropicrin. But is was thought possible, that such a condition might be obviated altogether by subjecting any organic compound liable to such decomposition, to the simultaneous action of both nascent chlorine and nitro group; and this has been found possible. Such a reagent has been found to be aqua regia. From a study of a large number of cases, it has been found that when aqua regia acts upon a substance-no matter if it does not contain any chlorine or nitro group-chloropicrin is invariably produced, provided the substance has a tendency to decompose destructively. The formation of chloropicrin fails under those cases in which the substance is quite stable in the presence of aqua regia and has no tendency to decompose. In those cases in which chloropicrin is formed, the yield of chloropicrin obtained varies. In some cases a quantitative yield of chloropicrin is obtained, in others it is produced in moderate quantity and again in some a trace of it is formed.

Again, the formation of chloranil has been studied in a very limited number of cases. Chloranil is formed by leading chlorine into an alcoholic solution of chlorisatin. ${ }^{2}$ It has been obtained by the action of a mixture of hydrochloric acid and potassium chlorate on aniline, phenol, ${ }^{3}$ salicylic acid, nitrosalicylic acid and dinitrosalicylic acid. ${ }^{4}$ It has also been obtained by the action of the same reagents on tyrosin ${ }^{5}$ and on $m$-aminobenzoic acid. ${ }^{6}$ Ty the interaction of fuming nitric acid on perchlorophenol, Merz and Weith ${ }^{7}$ obtained chloranil. Beilstein and Kurbatow ${ }^{8}$ obtained it from I,2,4,5-tetrachlorobenzene. Barral ${ }^{9}$ obtained chloranil from hexachlorocyclohexadinon by means of fuming nitric acid. Elbs and Brunnschweiber ${ }^{10}$ gave a method of its preparation from aniline, sodium

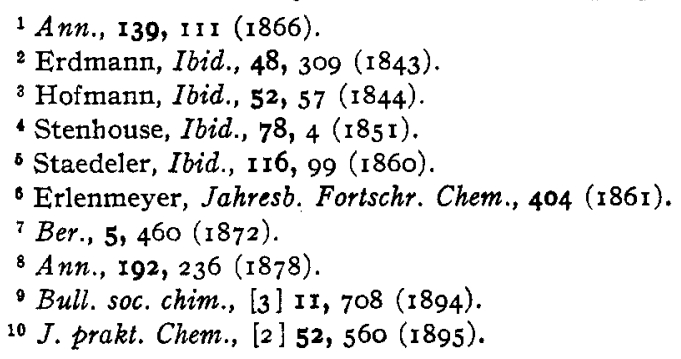


dichromate and hydrochloric acid. Witt and Toechemittler ${ }^{1}$ prepared chloranil by the treatment of 2 : 6-dichloro- $p$-phenylenediamine with potassium chlorate and hydrochloric acid. Chloranil is prepared by Graebe, ${ }^{2}$ from $p$-phenylenediamine with potassium chlorate and hydrochloric acid and oxidizing the product by means of acid dichromate. It is manufactured on the large scale by the treatment of 2,4,6-trichlorophenol with chromic acid mixture. Bouveault ${ }^{3}$ treats commercial chloranil with fuming nitric acid and potassium chlorate with the view of oxidizing the lower chloroquinones. One of $u^{4}$ has described reactions for the preparation of chloranil from $p$-phenylenediamine and hydroquinone by the action of aqua regia, which has been shown to be a good method for the preparation of chloranil.

It is evident from the above that chloranil has been prepared from a large number of sources and no generalization of their formation has as yet been noticed. It will be shown, from the study of a large number of cases, that the formation of chloroquinones is quite general in certain classes of compounds. By the action of aqua regia, which has a far greater oxidizing and chlorinating action than potassium chlorate and hydrochloric acid, on aromatic compounds, chloranil is very frequently formed; and as chloranil itself breaks up into chloropicrin by the action of aqua regia, this is also a general product of such reactions.

The essential condition of the formation of chloranil is that the aromatic compounds which are substituted in the p-positions, by particularly easily replaceable groups, yield tetrachloroquinone readily and with good yield, since the influence of side reaction is quite small in those cases. In the case of hydroxy and amino substituted products, chloranil is formed, but is accompanied by a large amount of tarry and other secondary products. In such cases the substance passes through a quinoid structure during the treatment with aqua regia. Again in those compounds which already possess simple or complex quinoid structures, chloranil is formed tolerably readily and in good yield. For instance, hydroquinone, which readily gives quinone on oxidation, yields tetrachloroquinone in almost quantitative yield by the action of aqua regia. Aniline black, which possesses a complex quinoid structure, yields chloranil easily. In the case of compounds which contain complex nuclei, such as naphthalene and anthracene derivatives, the formation of chloranil does not take place, the only effect is the formation of varying quantities of chloropicrin with considerable charring of the products. In the case of compounds which are otherwise substituted, considerable charring is the result with the formation of chloropicrin.

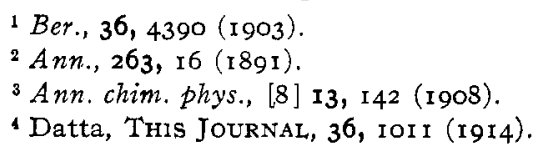


In a preliminary communication ${ }^{1}$ the action of aqua regia on acetone, ether, ethyl, methyl and allyl alcohols, acetic and formic acids have been studied and it has been shown that an almost quantitative yield of chloropicrin is formed in the case of acetone suitable for the preparation of this substance in the laboratory. The action of aqua regia on the following substances has been further studied:

\section{Aliphatic Compounds.}

Propyl Alcohol. $-5 \mathrm{cc}$. of propyl alcohol are added to $25 \mathrm{cc}$. of a mixture of nitric and hydrochloric acids $(1: 2)$ and the whole is warmed on the water bath for three to four hours. At the end of the operation, a very pungent odor of chloropicrin is noticed and at the same time a small quantity of an oil separated. The whole is subjected to steam distillation, when a colorless oil distils over and collects at the bottom of the aqueous layer. This is pure chloropicrin as is identified by its boiling point which is between II 2 to $113^{\circ}$.

0.2537 gave $0.647 \mathrm{I} \mathrm{AgCl}$; Calc. for $\mathrm{CCl}_{3} \mathrm{NO}_{2}: \mathrm{Cl}=64.02$. Found: $\mathrm{Cl}=63.10$.

Isopropyl Alcohol.-The decomposition of isopropyl alcohol to chloropicrin is almost quantitative, since its immediate oxidation product is acetone, which has been found to give a good yield of chloropicrin. Io cc. of isopropyl alcohol are added to $50 \mathrm{cc}$. of aqua regia, when an instantaneous vigorous reaction takes place with the separation of a large quantity of an oil. The reaction is completed by heating on the water bath. The product is distilled with steam and the oil collected, which, when dried and distilled, is found to be chloropicrin boiling at $112-113^{\circ}$.

0.1524 gave $0.3890 \mathrm{AgCl}$; Calc. for $\mathrm{CCl}_{3} \mathrm{NO}_{2}$ : $\mathrm{Cl}=64.02$. Found: 63.24 .

Butyl Alcohol.-io cc. of butyl alcohol are taken in a flask and to this about $50 \mathrm{cc}$. of a mixture of nitric and hydrochloric acids ( 112 ) are added and the whole is warmed on the water bath with a reflux for three hours. A colorless oil begins to collect at the bottom which increases in quantity as the operation goes on. The whole was subjected to steam distillation and identified as usual to be chloropicrin boiling between II $2-1 \mathrm{I} 3^{\circ}$.

Isobutyl Alcohol. $-5 \mathrm{cc}$. of isobutyl alcohol are added to $25 \mathrm{cc}$. of aqua regia. Only a slight reaction takes place in the cold, increasing somewhat on warming. After the end of the reaction no oil separates but a pungent smell of chloropicrin is produced, indicating that this compound is decomposed only partially.

Glycerol.- Io cc. of glycerol are taken in a flask fitted with a reflux condenser and to it $60 \mathrm{cc}$. of aqua regia are added. There is no reaction in the cold, but on warming on water bath a reaction seems to take place. The operation is continued for four hours. After the operation no oil separates, but a pungent smell of chloropicrin is noticed, which points to the fact that the chloropicrin which is formed only in small quantities is kept dissolved in dilute acids. The chloropicrin is secured by steam distillation and identified as usual.

Cane sugar gives a very small yield of chloropicrin on account of the fact that it is oxidized rapidly by the nitric acid to stable compounds such as oxalic acid. To $5 \mathrm{~g}$. of cane sugar in a flask, $50 \mathrm{cc}$. of aqua regia are added. No reaction takes place in the cold, but on warming on the sand-bath reaction goes on with the charring of the sugar. On continuing the operation for three to four hours, the solution which smells distinctly of chloropicrin ultimately turns yellow.

Maltose gives only a trace of chloropicrin when $5 \mathrm{~g}$. of maltose are heated on a reflux apparatus with $60 \mathrm{cc}$. of aqua regia. After the mixture is heated on the sand bath for

${ }^{1}$ Loc. cit. 
four to five hours a pale yellow solution is left which has only a faint smell of chloropicrin.

Grape sugar is warmed on the sand bath when a trace of chloropicrin is formed.

Starch is warmed on the sand bath with aqua regia for three hours. At the end of the operation only a slight smell of chloropicrin is noticed.

Methyl Ethyl Ketone.-On treating methyl ethyl ketone with aqua regia on the water bath, a complete decomposition of the ketone takes place and an almost quantitative yield of chloropicrin is the result, which is identified as usual.

Diethyl ketone decomposes on treatment with aqua regia and a good yield of chloropicrin is obtained.

Tartaric acid is heated on the sand bath with aqua regia for several hours. In this case, no smell of chloropicrin could be noticed. Hence the substance is quite stable in the presence of aqua regia and does not decompose destructively.

Chloroform.- Io cc. of chloroform are treated with $60 \mathrm{cc}$. of aqua regia. Noaction takes place in the cold, but it goes on slowly as it is heated on the water bath with reflux. At the end of the operation only a slight smell of chloropicrin could be noticed.

Chloral Hydrate. - When $5 \mathrm{~g}$. of chloral hydrate are treated with $30 \mathrm{cc}$. of aqua regia the reaction starts in the cold. The mixture is warmed on the water bath under reflux for three hours. At the end of the operation, a strong smell of chloropicrin is noticed.

Allyl Mustard Oil.-To $25 \mathrm{cc}$. of aqua regia, $4 \mathrm{cc}$. of allyl mustard oil are slowly added. At first no reaction takes place, but after a time a vigorous reaction ensues with the frothing up of the mixture. When the reaction has subsided, the whole is heated on the sand bath for two hours. At the end of the operation a pale yellow oil is found under the acid mixture. This is distilled in steam and the chloropicrin obtained is identified as usual.

Carbamide is heated under reflux with aqua regia for a good length of time. At the end only a slight smell of chloropicrin is noticed, pointing to the fact that urea is decomposed to a slight degree under the influence of aqua regia to form chloropicrin.

Guanidine carbonate is heated under a reflux on a sand bath with aqua regia. Only a trace of chloropicrin is formed which is recognized by its odor. It appears that the formation of stable salts hinders the decomposition of the compound to chloropicrin.

Hexamethylenetetramine is heated with an excess of aqua regia on the water bath for several hours. At the end of the operation, a colorless solution is obtained which smells only slightly of chloropicrin.

Uric acid is treated with aqua regia. At first no reaction takes place but after five minutes, bubbles of gas begin to rise, the substance going into solution gradually until it is completely dissolved. The mixture is heated on the sand bath for three to four hours. The resulting solution had a distinct smell of chloropicrin.

Theobromine on heating with the acid mixture yields a solution which smells slightly of chloropicrin.

Caffeine.-When the acid mixture is added to caffeine, a reaction takes place at once. The mixture is warmed on the sand bath for three to four hours. At the end the resulting solution smells distinctly of chloropicrin but no oil separates at the bottom. Hence the amount of chloropicrin formed is only minute.

\section{Aromatic Compounds.}

$p$-Aminophenol.-On adding $p$-aminophenol to a mixture of nitric and hydrochloric acids, at first there is no action, but soon afterwards the action commences with frothing. The mixture is then warmed on the water bath for two hours, and at the end of the operation a sulfur yellow crystalline product is produced. In this case no tarry 
product is formed. The yellow product obtained is crude chloranil which is purified by sublimation and is found to melt at $289^{\circ}$ in a sealed capillary tube.

0.162 I gave $0.3728 \mathrm{AgCl}$; Calc. for $\mathrm{C}_{6} \mathrm{Cl}_{4} \mathrm{O}_{2}: \mathrm{Cl}=57.14$. Found: 56.89 .

The supernatant liquid smells strongly of chloropicrin. Hence chloropicrin is formed at the same time, which is isolated and identified in the usual manner.

2,4-Diaminophenol.- This was heated under a reflux with aqua regia for three hours. The product consists of a yellowish liquid smelling strongly of chloropicrin and at the same time, a yellowish brown semisolid product is deposited underneath. It was filtered, washed and dried. On sublimation, the mass charred and a sublimate was obtained in beautiful yellow plates, melting at $289^{\circ}$, which is pure chloranil. In this case the yield is very small, as a considerable portion is converted to a tary mass with the formation of a large amount of chloropicrin.

$p$-Diaminodiphenylamine.-On treating the substance with aqua regia, at first very slight reaction takes place, which however is found to go on slowly on heating under reflux on a water bath. At the end of three hours, a brown solid substance is seen floating at the time, while the mixture smells strongly of chloropicrin. The operation is continued with a fresh quantity of aqua regia for some time. The brown product on sublimation gave a small yield of chloranil. Io $\mathrm{g}$. of the substance yielded $3 \mathrm{~g}$. of tetrachloroquinone.

Sulfanilic acid is treated with aqua regia when it begins to dissolve slowly. The mixture is then warmed on the water bath under reflux when within an hour, a yellowish crystalline product is seen to be formed on the surface of the acid mixture, which gradually increases in quantity during the progress of the reaction. After three hours the operation is found to be complete, and the product obtained consists of beautiful yellow crystalline scales which is nearly pure chloranil. It is purified by a single sublimation. The supernatant liquid smells strongly of chlotopicrin, pointing to the fact that a good quantity of sulfanilic acid is decomposed to chloropicrin.

p-Nitrophenol.-On heating $p$-nitrophenol with aqua regia for several hours, a sulfur yellow crystalline solid is obtained without any charring of product. The solution smells strongly of chloropicrin. On sublimation pure tetrachloroquinone is obtained melting at $289^{\circ}$.

p-Hydroxybenzoic Acid.-On heating p-hydroxybenzoic acid with aqua regia for several hours, a good yield of chloranil is obtained. This is unaccompanied by any tarry matter. The solution smells strongly of chloropicrin.

p-Toluidine.-When this is treated with aqua regia a reaction takes place at once. The mixture is warmed on the water bath when a dark oily substance is formed which deposits at the bottom. The liquid also smells strongly of chloropicrin. Upon prolonging the operation beautiful crystals are formed which are not chloroquinone. The product is under examination.

$p$-Cresol yields on prolonged treatment with aqua regia a smell of chloropicrin and a brown product is obtained which, however, does not yield any chloranil.

Aniline.-To aqua regia, aniline is added very gradually. At the end of the addition, a dark tarry product is seen floating on the acid mixture. The product is warmed on the water bath for six hours, during which the pungent smell of chloropicrin is noticed. As the operation is in progress the acid mixture is renewed. A brown pasty mass is obtained which yields a small quantity of tetrachloroquinone.

Diphenylamine.-To aqua regia, diphenylamine is gradually added when a reaction takes place, the solution turning dark blue. The product is warmed on the water bath with the renewal of the acid mixture, when a yellowish brown substance is obtained which gives a small yield of chloranil on sublimation. The mother liquid smells strongly of chloropicrin which is also formed in considerable quantity. 
2,4-Dichloroaniline.-On treating dichloroaniline with aqua regia, a reaction takes place in the cold. It is continued on the water bath and at the end a brown crystalline product is obtained which gave a fair yield of chloranil. The yield in this case is much greater than from aniline or diphenylamine. Chloropicrin is also formed at the same time.

Phenylhydrazine.-Phenylhydrazine hydrochloride is added to aqua regia and heated on the water bath. A reaction takes place with the evolution of heat, at the end of which, a tarry product is formed. This on sublimation gives a small yield of chloranil. During the operation the solution has a very pungent smell of chloropicrin.

Trichlorophenol.--This compound is heated in a reflux with aqua regia on the water bath. In the course of an hour, the transformation into chloranil is perceptible by the change of brown color of the product to bright yellow and generally becomes complete after three hours. A good yield of chloranil is obtained unaccompanied by any tarry matter. The mother liquor smells strongly of chloropicrin, a good quantity of which is formed at the same time.

Tribromophenol.-On treating tribromophenol with aqua regia on the water bath it is found that the bromine is replaced by chlorine and ultimately tetrachloroquinone is the result. A good quantity of chloropicrin is also formed at the same time.

Aniline Black.-Aniline black on prolonged treatment gradually turns from black to yellow. At the same time a pungent smell of chloropicrin is noticed. The yellow substance on sublimation gives a good yield of tetrachloroquinone.

Methyl orange on treatment with aqua regia, yields beautiful yellow crystals unaccompanied by any tarry matter. This is sublimed to get pure chloranil which is formed in good yield. The yellowish brown liquid smells strongly of chloropicrin.

Azobenzene gives a brown product, as also an abundant quantity of chloropicrin. In this case only a trace of tetrachloroquinone could be obtained.

$m$-Cresol on similar treatment yields a brown product from which a very small quantity of chloranil could be isolated, but chloropicrin is formed abundantly.

$m$-Phenylenediamine on treatment with aqua regia first yields a deep brown oily liquid which subsequently turns into a yellow crystalline solid. The yellow crystalline substance gives a fair yield of chloranil. The mother liquid strongly smells of chloropicrin.

$m$-Nitraniline yields a brown semi-solid product which gives a small quantity of chloroanil and an abundant quantity of chloropicrin.

$o$-Phenylenediamine on prolonged treatment yields a tarry mass which does not give tetrachloroquinone. In this case, no chloropicrin is formed.

o-Nitrophenol on treatment with aqua regia gradually passes from a dark colored pasty mass to a brown substance and thence to a yellow crystalline product in course of three hours. This yields a fair quantity of chloranil. A good quantity of chloropicrin is also formed at the same time.

$o$-Cresol.-Unlike $m$ - and $p$-cresols, $o$-cresol is found to give good quantities of chloranil and chloropicrin.

On treating 0 -cresol with aqua regia, a dark oily product is formed which is converted into a brown crystalline substance as the action is prolonged. This on sublimation gives chloranil. The mother liquor smells strongly of chloropicrin.

Benzidine.-With aqua regia, it yields a brown tarry solid which does not give any tetrachloroquinone. But in this case, a good quantity of chloropicrin is formed.

Benzenesulfonic Acid.-In this case no chloranil is formed but a good quantity of chloropicrin is formed.

Benzamide.-By the action of aqua regia on benzamide, no chloranil is formed but a yellowish white substance is formed which is under examination. In this case no chloropicrin is formed. 
Acetophenone yields only chloropicrin and no tetrachloroquinone.

Benzophenone yields only chloropicrin and no chloranil. An oily substance is formed which seems to decompose to chloropicrin and no sign of the formation of a solid could be noticed.

Resorcinol.-By prolonged treatment of resorcinol with aqua regia as usual, a good quantity of chloropicrin is produced and also a black pasty mass, which gradually diminishes in quantity as the reaction is continued being ultimately converted into chloropicrin. In this case no chloranil is formed.

Pyrogallic acid yields a good quantity of chloropicrin and a colorless crystalline product which is under investigation.

Tannic and Gallic Acids. - The yield of chloropicrin from tannic and gallic acids is considerable. No chloranil is formed in these cases. Compounds which have got many hydroxy groups generally break up destructively with the formation of chloropicrin.

Saccharine.-This gives only a slight smell of chloropicrin. It, however, gives no chloranil.

Pyridine does not give either chloranil or chloropicrin when treated with aqua regia with which it forms salts.

Quinoline, however, is found to give chloropicrin in a slight degree. When heated with aqua regia, showing that the reagent decomposes the quinoline ring only partially.

Alpha naphthol.-This substance yields a tarry product from which only a minute quantity of tetrachloroquinone could be obtained. An abundant quantity of chloropicrin is formed.

Beta naphthol.- In the case of $\beta$-naphthol, a black substance is formed and an abundant quantity of chloropicrin. From the black substance no chloranil could be obtained. The only effect of prolonged action is that the black substance is diminished with the formation of chloropicrin.

Mesitylene, cymene and styrolene all yield chloropicrin with the charring of products. Naphthalene and anthracene yield small quantities of chloropicrin.

\section{The Preparation of Chloranil.}

The preparation of chloranil by the action of aqua regia on hydroquinone and p-phenylenediamine has been described in a previous communication and it may be pointed out that they give the best yield of chloranil of any of the substances which have been treated with aqua regia. In fact this would form the best method for the preparation of chloranil on a commercial scale. The existing method of the manufacture of chloranil is the oxidation of trichlorophenol with potassium dichromate. It has been found that the yield could be increased and the product obtained purer by treating trichlorophenol with aqua regia. The next method of importance giving a good yield of chloranil is the treatment of sulfanilic acid with aqua regia. Sulfanilic acid which has amino and sulfonic groups in the p-positions is readily converted into chloranil, the sulfonic and amino groups being detached. The yield in this case is not so high as in the previous cases as in a good portion the molecule is ruptured with the formation of chloropicrin. In view of the fact that it is a cheap substance, this would serve as the most economical method of manufacture of chloranil on the large scale. The yields which have been obtained are as follows: 
From $20 \mathrm{~g}$. of hydroquinone and also of $p$-phenylenediamine I 3 to 14 g. of pure chloranil could be obtained; from $20 \mathrm{~g}$. trichlorophenol, a yield of $10 \mathrm{~g}$. could be realized and from $20 \mathrm{~g}$. of sulfanilic acid, a yield of 6-7 g. could be secured.

CAlCUTtA, INDIA.

[Contribution from the Defartment of Chfmistry of Bryn Mawr College.]

\section{THE REVERSIBLE REPLACEMENT OE ALCOHOLS IN ALDE- HYDEALCOHOLATES. ${ }^{1}$}

By Marguerite Willcox and Roger F. Brunel.

Received June 21, 1916.

\section{Introduction.}

Few systematic attempts have as yet been made to study the variation of the equilibrium in reactions of organic compounds with alteration of the constitution of one of the reacting substances. Such an investigation has been undertaken here with the hope of gaining a more definite knowledge of the relation existing between the structure of aliphatic compounds and their chemical affinity, and of determining what factors play an important part in governing this relationship.

Ostwald ${ }^{2}$ believed that the dissociation constants of acids, bases, and salts were determined by the constitution and composition of the substance, and could be considered as a measure of chemical affinity. He investigated the dissociation constants of organic acids at some length; but the relation between the affinity of organic acids, as expressed by their dissociation constants in solution, and their affinity, as exhibited in organic reactions, is by no means clear. In particular such attempts as that of Derick $^{3}$ to explain the reactions of all sorts of organic compounds by a consideration of ionization constants cannot be regarded as convincing.

Davis ${ }^{4}$ has recently investigated the effect of constitution on chemical affinity by studying the equilibria reached in the hydrolysis of various substituted formanilides into amines and formic acid. Since, however, his paper deals primarily with substitution in the benzene ring, the results obtained are not directly comparable with those given in this paper.

The influence which constitution exerts upon the velocity and limit of esterification of several classes of compounds, including saturated and

${ }^{1}$ Dissertation presented by Marguerite Willcox to the Faculty of Bryn Mawr College in partial fulfilment of the requirements for the degree of Doctor of Philosophy.

2 "Outlines of General Chemistry," p. 373 (r890). This subject has been extensively discussed in the chemical literature. Michael, J. prakt. Chem., N. S., 60, 333, made such data the original basis of his scale of combined influence. Among others who have discussed this subject Wegscheider is particularly to be mentioned Mon, 23, 287; 26, 1265; Z. Elektrochem., 18, 277, and in This Journal, Derick, 32, 1333 (1910).

3 This Journal, 32, 1333 (1910).

- Z. physik. Chem., 78, 353, 369 (1911). 